\title{
Review of Desloratadine Data Using the ARIA Guidelines
}

\author{
Elisa Villa, Anthi Rogkakou, Valentina Garelli, and G. Walter Canonica
}

Key Words: seasonal allergy rhinitis, persistent allergic rhinitis, ARIA, disease management, ACCEPT trials, H1-antihistamines

(WAO Journal 2012; 5:S6-S13)

D esloratadine is a second-generation, nonsedating H1antihistamine that first became available for the treatment of allergic rhinitis (AR) in 2001; it is currently approved for the treatment of AR irrespective of duration (intermittent/ persistent) or seasonality (seasonal/perennial) of symptoms. ${ }^{1}$ Its pharmacological characteristics are further described and underlined in a paper published in 2004: desloratadine is a powerful and selective $\mathrm{H}_{1}$ receptor blocker, without clinically relevant pharmacokinetic interference by foods, medications, or intestinal transport proteins, nor interference with cytochrome P 450 3A (CYP3A), and presenting additive antiallergic activities; these anti-inflammatory effects are related to the modulation of cytokines, adhesion molecules, and effector cells, such as eosinophils. ${ }^{2}$

From 2004 to 2009, several data concerning the use of desloratadine and its clinical efficacy in allergic respiratory diseases have been collected and published. The results about the desloratadine use in clinical trials are well known in both persistent and seasonal allergic rhinitis (PAR and SAR, according to previous classifications): the molecule has demonstrated to be effective in relieving nasal and ocular symptoms and improving nasal airflow and overall respiratory symptoms, also referred to a concomitant asthmatic condition (Table 1).

A paper of great interest has been published in 2007 by Canonica et $\mathrm{al}^{3}$ : it is a meta-analysis of double-blind, randomized, controlled clinical trials (performed between January1966 and May 2006) of desloratadine efficacy in the treatment of AR in adult patients. Through a search of MEDLINE,EMBASE,LILACS, and CINAHL databases, a total of 57 studies were collected and analyzed, and 13 randomized, double-blind, controlled trials were included in the metaanalysis. The studies showed a significant heterogeneity among their results because of the different study methodologies used. The analysis of total symptoms scores included 3108 patients: 1553 received desloratadine and 1555 placebo. The patients assuming desloratadine presented a significant reduction in total symptoms scores when compared with

\footnotetext{
From the Allergy and Respiratory Diseases, Department of Internal Medicine, University of Genoa, Genoa, Italy.

Correspondence to: G. Walter Canonica, MD, Allergy and Respiratory Diseases, DIMI, Department of Internal Medicine, University of Genoa, Largo Rosanna Benzi 10, Genoa 1-16132, Italy. E-mail: canonica@unige.it. Copyright (c) 2012 by World Allergy Organization
}

placebo (standardized mean difference [SMD] $-1.79 ; 95 \%$ CI -3.10 to $-0.47 ; P=0.008)$; in a parallel manner, total nasal symptoms score significantly decreased in patients treated with desloratadine versus placebo (SMD - 0.66; 95\% CI: -0.91 to $-0.42 ; P<0.001)$. Only in a very small population $(n=86)$, comparison with levocetirizine in terms of total nasal symptoms was possible, but no statistical difference between the 2 compounds was recorded (Table 2 ).

Concerning nasal airflow assessment (both after clinical treatment and in the allergen challenge setting), 438 patients from 7 studies were analyzed: 218 were treated with desloratadine and 220 with placebo. The difference between desloratadine and placebo resulted to be significantly in favor of desloratadine (SMD 0.32; 95\% CI: 0.10 to $0.55 ; P=0.005$ ).

In terms of nasal eosinophils, 4 studies comparing desloratadine and placebo were included, with a total number of 133 patients: desloratadine was significantly more effective than placebo (SMD $-1.28 ; 95 \% \mathrm{CI}-2.57$ to $0.01 ; P=0.05)$.

In conclusion, for the first time this meta-analysis demonstrates that the efficacy of desloratadine versus placebo in $\mathrm{AR}$ is supported by Ia evidence, the highest ranking in evidence-based medicine. Furthermore, this is the first meta analysis of clinical data regarding an antihistamine that shows significant beneficial effects not only on clinical symptoms but also objective measures of nasal blockage and allergic inflammation. The overall results support evidence of desloratadine efficacy in terms of improving nasal airflow more than placebo and confirm that nasal symptomatic benefits are accompanied by an objective decrease in nasal obstruction, representing the main clinical feature of AR.

Focusing on intermittent allergic rhinitis (IAR), we have to remember the ARIA document published in 20014; the classification in 4 categories (mild and moderate/severe intermittent; mild and moderate/severe persistent, depending on severity and duration of symptoms and quality of life) has been recently confirmed ${ }^{5}$ and a stepwise pharmacologic treatment is proposed on the basis of ARIA clinical categories. Recently, the authors of the last edition noted that 2 RCTs with oral $\mathrm{H}_{1}$-antihistamines have been carried out in PER, ${ }^{6,7}$ but no randomized controlled trials (RCTs) in IAR was published, although one was incorrectly ascribed to IAR. ${ }^{8}$

Desloratadine is a valid therapeutic tool for allergic rhinitis, in terms of both efficacy and safety: different trials showed that it improves symptoms and quality of life in seasonal $^{3,8,9}$ and perennial allergic rhinitis, ${ }^{10,11}$ referring to the previous classification. However, it has not been evaluated yet in IAR or PER as defined in the latest ARIA document.

ACCEPT-1 (Aerius Control: Clinical and Evaluative Profile of Treatment) is the first study to test both efficacy and 
TABLE 1. Recent Data About Desloratadine Use in Respiratory Diseases: Clinical Trials and End Points

\begin{tabular}{lll}
\hline Criteria & \multicolumn{1}{c}{ Data } & \multicolumn{1}{c}{ End Point } \\
\hline SAR & Pradalier et al. Allergy. 2007;62:1331 (AERONEF) & TSS \\
& Kosa L. JACI. 2008;121: S51 (open label) & Nasal, ocular \\
& Keith et al. Clin Ther. 2007;121;1151-1155 & Nasal, ocular, respiratory, and overall symptoms \\
& Meltzer et al. Ann Allergy Asthma Immunol. & TSS, nasal airflow \\
& 2006;96;363-368. & TSS; PNIF \\
PAR & Kim et al. Ann Allergy Asthma Immunol. & \\
& 2006;96:460 (TNSS, PNIF) & TNSS, asthma symptoms in peds \\
IAR/PER & ACCEPT & TSS, nasal airflow \\
Asthma & Rossi et al. Allergy. 2005; 60:416. & SAR, PAR, TSS \\
Meta-analysis & Canonica et al. Allergy. 2007;62:359. & TNSS, asthma symptoms in peds \\
Peds & Tassinari et al. AAAAI 2006. (SAR/PAR TSS) & PRN use in peds \\
& Rossi et al. Allergy. 2005; 60:416-417 & \\
\hline
\end{tabular}

TABLE 2. Comparisons of Drug Outcome for Studies Included in the Meta-Analysis

\begin{tabular}{|c|c|c|c|c|c|c|c|}
\hline Outcome & Comparison & Studies $(n)$ & Patients $(n)$ & I $2(\%)$ & Effect & $95 \%$ CI & $P$ \\
\hline \multirow[t]{2}{*}{ Total symptom score } & DL-placebo & 11 & 3108 & 99.3 & -1.63 & -2.75 to -0.51 & 0.004 \\
\hline & DL-LCZ & 3 & 86 & 59.9 & 0.54 & -0.20 to 1.27 & 0.15 \\
\hline Total nasal symptom score & DL-placebo & 7 & 2883 & 86.8 & -0.66 & -0.91 to -0.42 & $<0.001$ \\
\hline Nasal airflow & DL-placebo & 5 & 438 & 18.1 & 0.32 & 0.10 to 0.55 & 0.005 \\
\hline \multirow[t]{2}{*}{ Nasal eosinophils } & DL-placebo & 4 & 133 & 89.7 & -1.28 & -2.57 to 0.01 & 0.05 \\
\hline & DL-LCZ & 3 & 88 & 93 & 1.37 & -0.74 to 3.49 & 0.20 \\
\hline \multirow[t]{2}{*}{ Nasal interleukin-4 } & DL-placebo & 3 & 88 & 94.5 & -2.18 & -5.01 to 0.64 & 0.13 \\
\hline & DL-LCZ & 3 & 88 & 90.2 & -0.66 & -0.91 to -0.42 & 0.08 \\
\hline
\end{tabular}

LCZ, levocetirizine; I2, I-square statistic; DL, desloratadine.

safety of desloratadine in patients with IAR as defined by ARIA criteria. ${ }^{12}$ The study was conducted in collaboration with GA ${ }^{2}$ LEN (Global Allergy and Asthma European Network), a consortium of the most important European research centers specializing in allergic diseases, and was a multicenter, multinational, randomized, double-blind, placebocontrolled, parallel-group phase IV study of 5-mg desloratadine (orally administered in the morning). The trial, carried out from September 5, 2006 to November 21, 2007, involved 60 centers in 15 different countries (Belgium, Canada, Denmark, Finland, France, Germany, Greece, Hungary, Italy, The Netherlands, Portugal, Russia, Spain, Sweden, and Turkey).

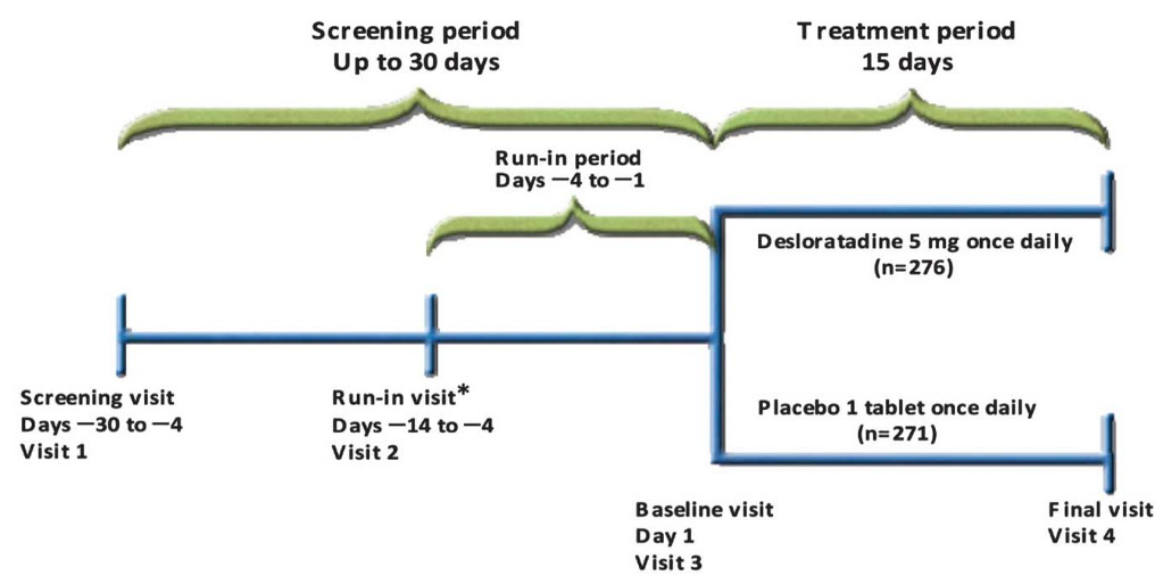

*If no medication washout is needed, visit 1 and visit 2 may be combined. 
TABLE 3. Primary, Secondary, and Exploratory Outcome Variables (Intent-to-Treat Analysis)

\begin{tabular}{|c|c|c|c|c|c|}
\hline & \multicolumn{2}{|c|}{ 5-mg DL } & \multicolumn{2}{|c|}{ Placebo } & \multirow[b]{2}{*}{$\boldsymbol{P}$} \\
\hline & $\begin{array}{l}\text { Baseline LS } \\
(\mathrm{m} \pm \text { SEM) }\end{array}$ & $\begin{array}{c}\text { Days 1-15 LS } \\
(\mathrm{m} \pm \mathrm{SEM})\end{array}$ & $\begin{array}{l}\text { Baseline LS } \\
(\mathrm{m} \pm \mathrm{SEM})\end{array}$ & $\begin{array}{c}\text { Days 1-15 LS } \\
(\mathrm{m} \pm \mathrm{SEM})\end{array}$ & \\
\hline AM/PM T5SS reflective & $8.71 \pm 0.15$ & $-3.19 \pm 0.22$ & $8.49 \pm 0.15$ & $-2.29 \pm 0.22$ & $<0.001$ \\
\hline RQLQ total score (at end point) & $2.96 \pm 0.08$ & $-1.10 \pm 0.10$ & $2.80 \pm 0.08$ & $-0.73 \pm 0.10$ & $<0.001$ \\
\hline AM/PM T5SS instantaneous & $8.30 \pm 0.17$ & $-2.86 \pm 0.20$ & $8.17 \pm 0.17$ & $-1.90 \pm 0.20$ & $<0.001$ \\
\hline AM/PM rhinorrhea reflective & $1.81 \pm-0.04$ & $-0.58 \pm 0.05$ & $1.75 \pm 0.04$ & $-0.38 \pm 0.05$ & $<0.001$ \\
\hline AM/PM nasal congestion reflective & $1.96 \pm 0.04$ & $-0.56 \pm 0.05$ & $1.87 \pm 0.04$ & $-0.43 \pm 0.05$ & 0.013 \\
\hline $\mathrm{AM} / \mathrm{PM}$ sneezing reflective & $1.65 \pm 0.04$ & $-0.64 \pm 0.05$ & $1.61 \pm 0.04$ & $-0.42 \pm 0.05$ & $<0.011$ \\
\hline $\mathrm{AM} / \mathrm{PM}$ nasal itching reflective & $1.7 \pm 0.04$ & $-0.67 \pm 0.05$ & $1.65 \pm 0.04$ & $-0.43 \pm 0.05$ & $<0.001$ \\
\hline $\mathrm{AM} / \mathrm{PM}$ eye itching reflective & $1.38 \pm 0.06$ & $-0.58 \pm 0.05$ & $1.45 \pm 0.06$ & $-0.46 \pm 0.05$ & 0.047 \\
\hline AM/PM rhinorrhea instantaneous & $1.76 \pm 0.05$ & $-0.54 \pm 0.05$ & $1.72 \pm 0.05$ & $-0.33 \pm 0.05$ & $<0.001$ \\
\hline AM/PM nasal congestion instantaneous & $1.94 \pm 0.05$ & $-0.52 \pm 0.05$ & $1.87 \pm 0.05$ & $-0.39 \pm 0.05$ & 0.009 \\
\hline $\mathrm{AM} / \mathrm{PM}$ sneezing instantaneous & $1.56 \pm 0.05$ & $-0.59 \pm 0.05$ & $1.51 \pm 0.05$ & $-0.36 \pm 0.05$ & $<0.001$ \\
\hline AM/PM nasal itching instantaneous & $1.65 \pm 0.05$ & $-0.63 \pm 0.05$ & $1.61 \pm 0.05$ & $-0.37 \pm 0.05$ & $<0.001$ \\
\hline AM/PM eye itching instantaneous & $1.38 \pm 0.06$ & $-0.58 \pm 0.05$ & $1.46 \pm 0.06$ & $-0.45 \pm 0.05$ & 0.023 \\
\hline Sleep interference & $1.37 \pm 0.06$ & $-0.39 \pm 0.05$ & $1.38 \pm 0.06$ & $-0.27 \pm 0.05$ & 0.039 \\
\hline Activity interference & $1.72 \pm 0.05$ & $-0.60 \pm 0.06$ & $1.66 \pm 0.05$ & $-0.40 \pm 0.06$ & $<0.001$ \\
\hline Symptom severity reflective (VAS) & $57.4 \pm 1.36$ & $-17.2 \pm 1.50$ & $56.76 \pm 1.35$ & $-10.9 \pm 1.49$ & $<0.001$ \\
\hline Patient's evaluation of response WPAI-AS & NA & $3.24 \pm-0.10$ & NA & $3.66 \pm-0.10$ & $<0.001$ \\
\hline overall work impairment & $46.38 \pm 2.35$ & $-15.0 \pm 2.78$ & $41.37 \pm 2.27$ & $-5.7 \pm 2.69$ & 0.002 \\
\hline activity impairment & $48.24 \pm 1.85$ & $-15.3 \pm 2.20$ & $46.04 \pm 1.87$ & $-9.2 \pm 2.22$ & 0.007 \\
\hline
\end{tabular}

The patients eligible for the study were patients 12 years of age or older, with at least a 2-year history of allergic rhinitis symptoms defined according to the International Consensus on Rhinitis ${ }^{13}$; all the patients had to meet the ARIA criteria for IAR (symptoms of allergic rhinitis present $<4$ days per week or for $<4$ consecutive weeks per year) and present mild to moderate symptoms. The day of inclusion, the "total score of 5 symptoms" (T5SS) had to be at least 6; the sum of the daily averages of the diary recordings of the 12-hour morning (AM) plus evening (PM) T5SS collected for 4 days and the AM reflective T5SS on the morning of the randomization had to be 30 at least. Allergic sensitization was defined by positive skin prick tests to common aeroallergens carried out according to the $\mathrm{GA}^{2} \mathrm{LEN}$ skin test study. ${ }^{14} \mathrm{~A}$ washout period for any medication for allergic rhinitis was necessary 14 days before the patients' randomization (Fig. 1).

Validated methods were used to enroll patients. The primary outcome was the recommended T5SS: nasal congestion stuffiness, sneezing, rhinorrhea/nasal discharge, nasal pruritus, and eye itching. Each sign/symptom was scored 0 to 3 (none $=0$ to $3=$ severe) and recorded in personal patients' diaries, in the morning (AM) within 1 hour of awakening and in the evening (PM), approximately 12 hours later. The T5SS is the sum of the ratings for the individual scores.

The other outcomes considered were as follows:

- Symptom severity visual analog scale (VAS) assessment was recorded each morning within 1 hour of awakening and before dosing (AM) at the baseline visit and for each day of study treatment. Scores had a range from $0 \mathrm{~mm}$ (not at all bothersome) to $100 \mathrm{~mm}$ (very bothersome). ${ }^{15,16}$
- Patient's evaluation of therapeutic response to treatment was assessed by the patient at the final visit (day 15). The evaluation considered the whole time period since the baseline up to the final visit, compared with baseline. The score followed a 5-point scale: complete relief, marked relief, moderate relief, slight relief, and no relief.

- Rhinoconjunctivitis quality of life questionnairestandardized version (RQLQ-S) was completed by the patient at baseline and final visits in patients $\geq 18$ years of age. RQLQ ${ }^{16}$ was a key secondary outcome measure.

- Interference with sleep and with activities of daily living was assessed. At the run-in visit, during the run-in period (days -4 to -1 ), and continuing through the final visit (day 15: visit 4), patients recorded in their daily diaries the 2 interference rating scores: AM evaluations of interference with sleep caused by allergic rhinitis symptoms and PM evaluations of interference with daily activities determined by allergic rhinitis symptoms during the same day.

- The allergic rhinitis specific work productivity and activity impairment questionnaire (WPAI-AS) was completed by the patients at the baseline and through the final visit. ${ }^{17,18}$

- Study drug compliance with study drug was evaluated by counting and comparing the number of tablets dispensed at the baseline visit with the number returned at the final visit.

Instantaneous T5SS, individual symptom scores, VAS assessments, ${ }^{15,16}$ and patient's evaluations of response were considered as secondary end points; WPAI-AS ${ }^{17,18}$ were used as exploratory outcome measures. 
A total of 262 patients treated with desloratadine (the active group) and 256 individuals treated with placebo completed the study.

The results clearly show that desloratadine provided a 24-hour efficacy, as already known, significant at day 1 and lasting throughout the study. Nevertheless, the maximum effect was registered after 1 week of treatment for the primary outcome measure; it is possible that the intermittent nature of the symptoms could have a role in attenuating the treatment differences between the 2 groups. The efficacy of desloratadine was found for both instantaneous and reflective symptoms. A $37.7 \%$ change in T5SS reflective in the desloratadine group and a $27.8 \%$ change in the placebo group $(P<0.001)$ were recorded.

For VAS, a significant difference was demonstrated at day 1 and continued throughout the study. The mean baseline VAS ratings were 57.4 in the desloratadine group and 56.7 in the placebo group: the patients mostly presented moderate to severe symptoms. At the end of the study, patients in the desloratadine group showed a significantly greater improvement in VAS rating compared with placebo (Table 3, Fig. 2).

RQLQ was significantly improved in the active group with respect to the placebo group. Mean total RQLQ-S score at baseline was 2.96 in the desloratadine group and 2.80 in the placebo group, thus confirming that patients presented moderate to severe IAR; at the study end, total RQLQ global score was significantly improved. All RQLQ evaluations, except for sleep, were significantly improved in the desloratadine group, if compared with placebo. Globally, all the secondary and exploratory outcomes were also significantly improved by desloratadine.

In addition, all nasal symptoms were significantly improved in the patients treated with desloratadine, compared with placebo. Nasal congestion relief probably is strictly related to sleep scores improvement.

The symptoms related to AR may determine a considerable impairment of subjective quality of life, in terms of physical and emotional comfort and functional capacity, with consequent fatigue, absenteeism, and poor task performance. ${ }^{19}$ The ACCEPT-1 is also the first study to provide that clinical improvement with desloratadine treatment has an economically relevant impact on the productivity of patients with IAR. Through the objective WPAI-AS assessment, for the first time a significantly greater increase in work and school productivity and improvement of daily activity was evidenced in patients treated with desloratadine with respect to placebo. In the United States, AR has been estimated to be responsible for about 2 million school days missed and 3.5 million lost work days annually, with consequent decreased productivity and a large economical impact. ${ }^{20}$

In addition, adverse events were comparable in the patients of the 2 groups: desloratadine and placebo was well tolerated and the rate of severe adverse events was identical and very low in the 2 groups. No life-threatening adverse event was recorded; only 4 adverse events in each group led to a stop in treatment.

The efficacy of desloratadine according to ARIA classification was conducted not only in patients affected by IAR but also in those affected by PER.
A Day

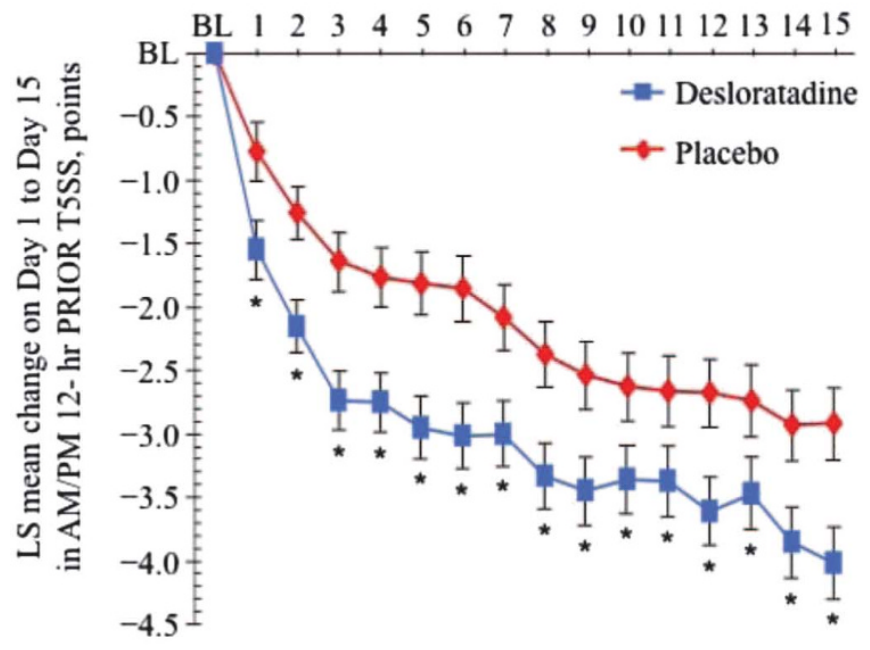

B Day

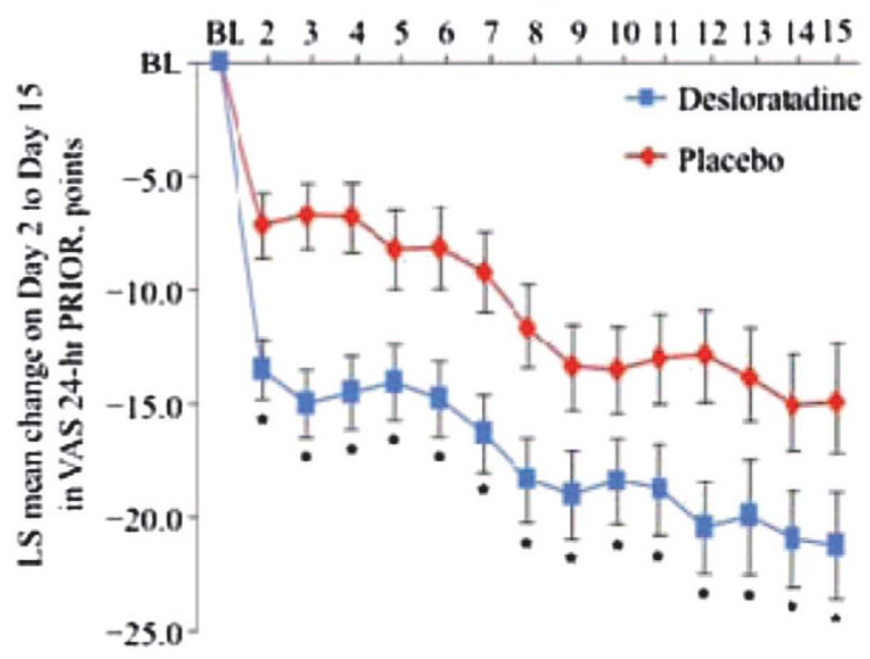

FIGURE 2. Evolution of reflective T5SS (a) and symptom severity by VAS (b).

According to the ARIA guidelines, PER is present $>4$ days per week and for $>4$ consecutive weeks per year. ${ }^{15}$ This innovative classification is closer to real life and takes into consideration patients' quality of life. Quality-of-life (QoL) outcomes are an important way to measure the negative effects of AR on patients in a way that is not limited to assessments of only sign and symptom severity, but also includes effects of the disease and treatment on the patient. In the study of persistent allergic rhinitis, the RQLQ is the most frequently used tool and has been validated in many studies and in different languages. The RQLQ-S provides information on the impact of AR and allergic conjunctivitis on a variety of domains that include emotions, practical problems, activities, and also nasal and other symptoms.

Desloratadine is a second-generation nonsedating antihistamine with both antihistaminic and anti-inflammatory properties. ${ }^{21}$ Desloratadine (DL) is effective and safe in the 
treatment of persistent allergic rhinitis. There is Ia evidence according to a meta-analysis of the literature about DL efficacy to improve nasal airflow, total symptoms and total nasal symptoms. $^{22}$

Although desloratadine has been shown to be safe and effective in the treatment of seasonal and perennial AR, ACCEPT $-2^{23}$ was the first prospective study of desloratadine in ARIA-defined PER. It has a similar study design to ACCEPT-1, previously conducted for intermittent allergic rhinitis.

The aim of the study was to evaluate the safety and the efficacy of desloratadine in this population. This multicenter, multinational, randomized, double-blind, placebo-controlled, parallel-group phase IV study of DL (5 mg, once daily, in the morning) was conducted with the collaboration of $\mathrm{GA}^{2} \mathrm{LEN}$ research centers at 83 sites in 15 countries. ${ }^{22}$ The study report conformed to the CONSORT (CONsolidated Standards of Reporting Trials) Statement. ${ }^{24}$

Patients aged 12 years or older had to have at least a 2year history consistent with symptoms of allergic rhinitis defined according to the International Consensus on Rhinitis ${ }^{3}$ and PER with moderate to severe symptoms according to the ARIA classification ${ }^{15}$ to be included.

At the inclusion (the start of the run-in period) the T5SS had to reach a score of 8 at least. The sum of the daily averages of the diary recordings of the 12-hour AM plus PM reflective T5SS collected for 4 days and the AM reflective T5SS on the morning of randomization had to be $\geq 40$.

The study design provided a run-in period lasting from 4 to 14 days. All the patients enrolled were randomized to receive 5-mg DL or placebo for 12 weeks. Visits took place on day 1 (baseline visit) and days 15, 29, 43, 57, and 85 after randomization during the treatment period (Fig. 3 ).

Rescue medications, such as cromoglycate (nasal and/ or ocular), were allowed after the first 4 weeks at the minimum dose required to control symptoms.
The primary objective was to compare the efficacy and safety of 5-mg desloratadine once daily versus placebo in patients with PER and the primary end point was the reflective T5SS in the intent-to-treat population from day 1 to day 29. The first key secondary end point was the mean change from baseline in RQLQ-S ${ }^{11}$ after 29 days of treatment. The other key secondary end point was the change from baseline in the 12-hour AM/PM reflective T5SS averaged during the 12 weeks of treatment (day 1 to day 85).

The instantaneous T5SS (days 1-29 and days 1-85), individual symptom scores (days 1-29 and days 1-85), VAS levels (days 1-29 and days 1-85), ${ }^{25,26}$ RQLQ (days 1-85), and patients' assessment of response (days 29 and $85)$ were considered as secondary end points. The WPAIAS (days 29 and 85$)^{27,14}$ was an exploratory outcome measure.

Adverse events were recorded at each visit on the case report form. The 931 patients were screened and 716 of them were randomized in the clinical trial. A total of 301 patients treated with DL and 261 patients receiving placebo completed the study.

The mean reduction from baseline in T5SS achieved with desloratadine during days 1-29 (3.76 points) was significantly greater if compared with placebo. The study also met this key secondary end point, with a statistically significantly greater decrease from baseline in mean AM/PM PRIOR T5SS across days 1-85 as compared with placebo. Similarly, these results achieved with desloratadine during the first 2 weeks and the first month of the study were significantly greater than placebo. Notably, this effect had an early onset of action, being seen as early as day 1 of the study. The significantly greater effect of desloratadine on T5SS lasted through the end of the 24-hour dosing interval, as shown here using the AM NOW T5SS scores. The 24-hour duration of effect of desloratadine was significantly longer than placebo from day 3 of

Treatment period

12 weeks

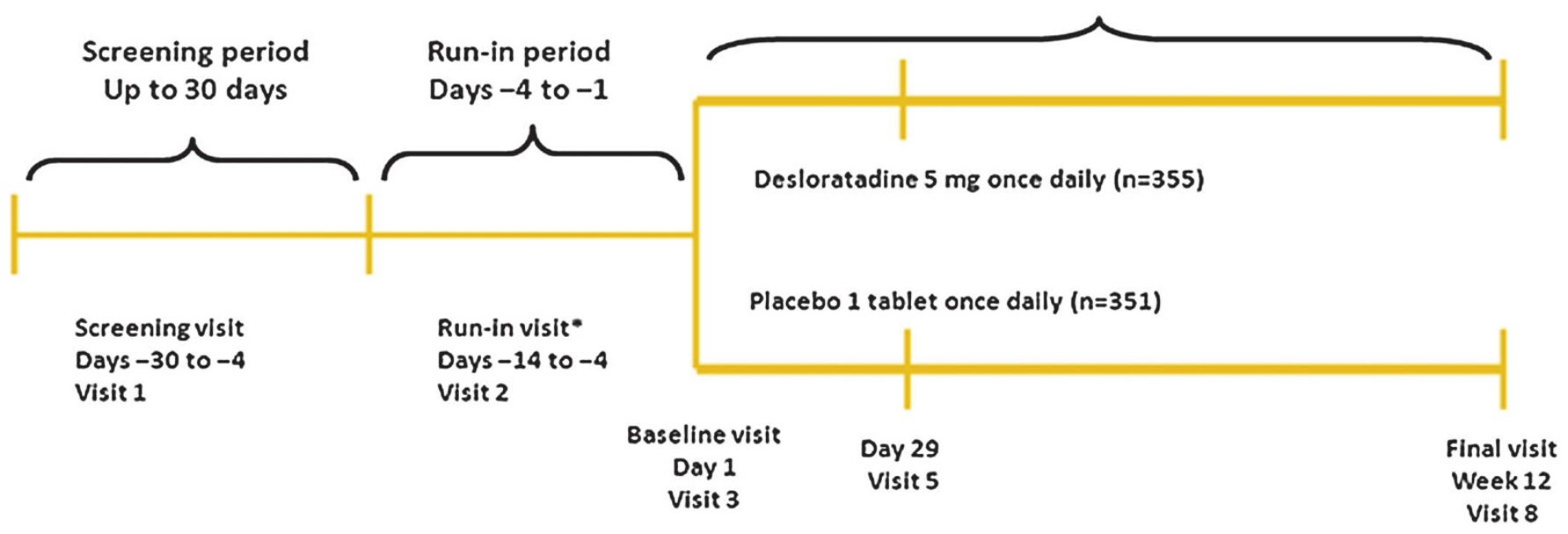

FIGURE 3. ACCEPT-2 study design 
A

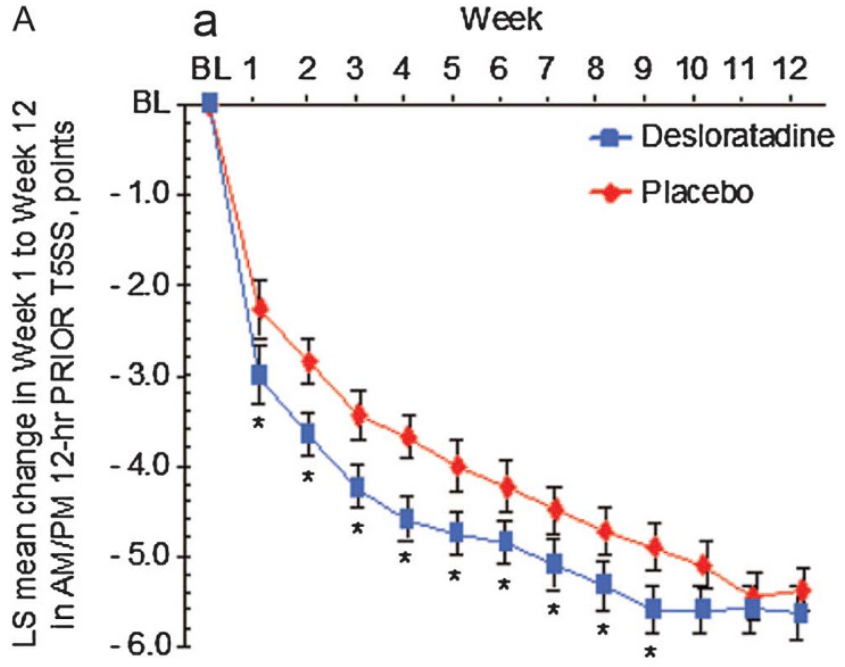

B

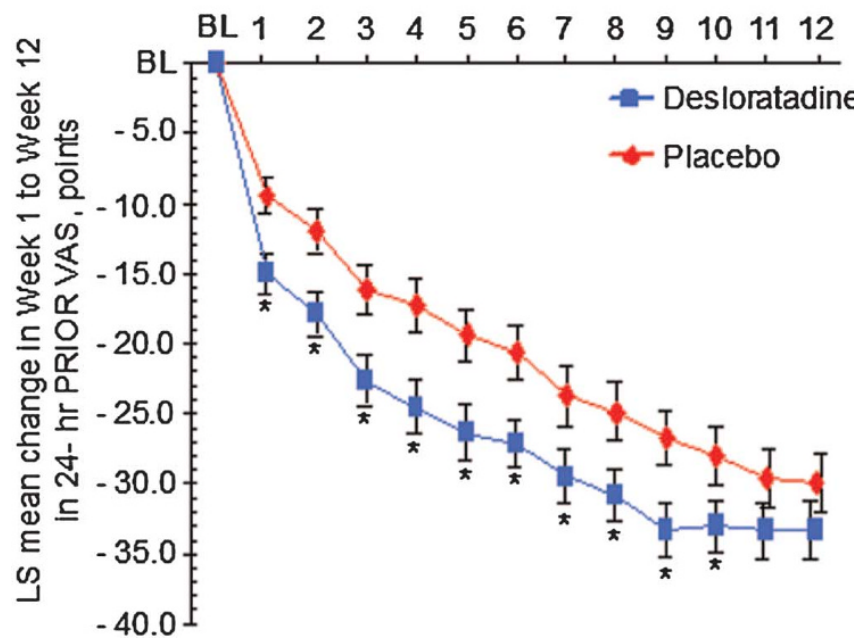

FIGURE 4. Evolution of reflective T5SS (a) and symptom severity by VAS (b). Least significant (LS) means obtained from an ANOVA model with treatment and site as a covariate in the model. ${ }^{*} P \leq 0.018$.

the study and endured across the full 12-week duration for days $2-85$.

Nasal congestion is a cardinal symptom of allergic rhinitis, difficult to treat and bothersome for the patient. Desloratadine has been shown previously to have a consistently beneficial effect on nasal congestion in seasonal/ perennial allergic rhinitis, but had not previously been studied in the setting of ARIA-defined PER.

Similarly, the total RQLQ-S score was significantly improved from baseline in the desloratadine group versus placebo at the end of the first month of the study, an effect that lasted through to the end of the study at day 85 (12 weeks). When the individual domains that constitute the RQLQ-S were assessed on their own, apart from non-nose/ eye symptoms, desloratadine treatment was shown to lead to a statistically significantly greater improvement in QOL as compared with placebo at the end point analysis. Significant differences between the active and the control group were registered with a $P<0.038$ for the following domains: activity, sleep, practical problems, nasal symptoms, eye symptoms, and emotion.

In previous studies about the use of desloratadine in the treatment of patients with seasonal/perennial allergic rhinitis, desloratadine has been shown repeatedly to reduce rhinitisinduced impairment of sleep and daily activities. To date, these effects of desloratadine had not been studied in thesetting of ARIA-defined PER.

As early as day 1 and for the whole duration of the study, desloratadine treatment was associated with a statistically significantly greater reduction in PER-induced interference with daily activities as compared with placebo. Similarly, desloratadine led to a statistically significant beneficial effect in improving PER-induced sleep disturbance during the first month of the study and across the 12-week duration of the study.

Allergic rhinitis is a large burden for the patient because of impairment of sleep/daily activities and reduced QoL. The statistically significant effect of desloratadine in reducing the burden of allergic rhinitis was measured using the new VAS tool. Desloratadine significantly decreased the VAS severity $(0=$ not bothersome, $100=$ very bothersome) from baseline from the first study time point (day 2 for VAS), which progressively improved, remaining statistically superior to placebo during the 12-week course of the study (Fig. 4, Table 4). Desloratadine was well tolerated and no life-threatening adverse events occurred.

In patients with intermittent (ACCEPT-1) and persistent (ACCEPT-2) allergic rhinitis, therapy with desloratadine provides a rapid, effective, and sustained relief of total and individual symptom scores, including nasal congestion. DL proved to have a 24-hour efficacy with significant improvement in daily activities and sleep, overall quality of life, work, or school productivity. DL provides a good safety and tolerability profile and does not present anticholinergic activity, sedative effects, or cognitive or psychomotor impairment. Desloratadine thus satisfies all the ARIA/EAACI criteria ${ }^{16,18}$ and is highly recommended as first-line treatment in both intermittent and persistent allergic rhinitis.

\section{ACKNOWLEDGMENTS}

The contents of this article were presented as an invited World Allergy Organization Lecture at the First Middle East Asia Allergy Asthma and Immunology Congress (MEAAAIC) in Dubai, UAE, March 26-29, 2009, as part of the symposium, "Current Concepts in Allergic Rhinitis, Rhinosinusitis and New Developments in Histamine-mediated Diseases."

Schering-Plough provided an educational grant for the symposium. 
TABLE 4. Primary, Secondary Outcome Variables (Intent-to-Treat Analysis)

\begin{tabular}{|c|c|c|c|c|c|}
\hline & \multicolumn{2}{|c|}{ 5-mg DL } & \multicolumn{2}{|c|}{ Placebo } & \multirow[b]{2}{*}{$P$} \\
\hline & $\begin{array}{l}\text { Baseline LS } \\
(\mathrm{m} \pm \text { SEM) }\end{array}$ & $\begin{array}{c}\text { LS } \\
(\mathrm{m} \pm \mathrm{SEM})\end{array}$ & $\begin{array}{l}\text { Baseline LS } \\
(\mathrm{m} \pm \mathrm{SEM})\end{array}$ & $\begin{array}{c}\text { LS } \\
(\mathrm{m} \pm \mathrm{SEM})\end{array}$ & \\
\hline AM/PM T5SS reflective (days 1-29) & $9.63 \pm 0.13$ & $-3.76 \pm 0.22$ & $9.55 \pm 0.21$ & $-2.87 \pm 0.21$ & $<0.001$ \\
\hline \multicolumn{6}{|l|}{ RQLQ total score } \\
\hline day 29 & $3.30 \pm 10.08$ & $-1.35 \pm 0.10$ & $3.15 \pm 0.08$ & $-0.95 \pm 0.10$ & $<0.001$ \\
\hline day 85 & & $-1.66 \pm 0.11$ & & $-1.39 \pm 0.12$ & 0.023 \\
\hline $\begin{array}{l}\text { AM/PM T5SS reflective } \\
\text { (days } 1-85 \text { ) }\end{array}$ & & $-4.50 \pm 0.23$ & & $-3.61 \pm 0.23$ & $<0.001$ \\
\hline \multicolumn{6}{|l|}{ AM/PM T5SS instantaneous } \\
\hline days $1-29$ & $9.35 \pm 0.15$ & $-3.41 \pm 0.22$ & $9.23 \pm 0.14$ & $-2.52 \pm 0.22$ & $<0.001$ \\
\hline days $1-85$ & & $-4.11 \pm 0.24$ & & $-3.22 \pm 0.23$ & $<0.001$ \\
\hline \multicolumn{6}{|l|}{$\mathrm{AM} / \mathrm{PM}$ rhinorrhea reflective } \\
\hline days $1-29$ & $2.10 \pm 0.04$ & $-0.81 \pm 0.05$ & $2.08 \pm 0.04$ & $-0.62 \pm 0.05$ & $<0.001$ \\
\hline days $1-85$ & & $-0.98 \pm 0.05$ & & $-0.77 \pm 0.05$ & $<0.001$ \\
\hline \multicolumn{6}{|l|}{ AM/PM nasal congestion reflective } \\
\hline days $1-29$ & $2.13 \pm 0.04$ & $-0.69 \pm 0.05$ & $2.1 \pm 0.04$ & $-0.53 \pm 0.05$ & 0.002 \\
\hline days $1-85$ & & $-0.87 \pm 0.05$ & & $-0.70 \pm 0.05$ & 0.002 \\
\hline \multicolumn{6}{|l|}{$\mathrm{AM} / \mathrm{PM}$ sneezing reflective } \\
\hline days $1-29$ & $1.88 \pm 0.04$ & $-0.83 \pm 0.05$ & $1.84 \pm 0.04$ & $-0.62 \pm 0.05$ & $<0.001$ \\
\hline days $1-85$ & & $-0.96 \pm 0.05$ & & $-0.76 \pm 0.05$ & $<0.001$ \\
\hline \multicolumn{6}{|l|}{$\mathrm{AM} / \mathrm{PM}$ nasal itching reflective } \\
\hline days $1-29$ & $1.89 \pm 0.04$ & $-0.77 \pm 0.05$ & $1.88 \pm 0.04$ & $-0.58 \pm 0.05$ & $<0.001$ \\
\hline days $1-85$ & & $-0.91 \pm 0.05$ & & $-0.65 \pm 0.05$ & 0.002 \\
\hline \multicolumn{6}{|l|}{$\mathrm{AM} / \mathrm{PM}$ eye itching reflective } \\
\hline days $1-29$ & $1.62 \pm 0.05$ & $-0.67 \pm 0.05$ & $1.64 \pm 0.05$ & $-0.52 \pm 0.05$ & 0.006 \\
\hline days $1-85$ & & $-0.78 \pm 0.05$ & & $-0.65 \pm 0.05$ & 0.016 \\
\hline \multicolumn{6}{|l|}{ Sleep interference } \\
\hline $2-29$ days & $1.58 \pm 0.05$ & $-0.54 \pm 0.06$ & $1.59 \pm 0.05$ & $-0.42 \pm 0.05$ & 0.022 \\
\hline $2-85$ days & & $-0.65 \pm 0.06$ & & $-0.52 \pm 0.06$ & 0.023 \\
\hline \multicolumn{6}{|l|}{ Activity interference } \\
\hline $1-29$ days & $1.89 \pm 0.04$ & $-0.69 \pm 0.05$ & $1.89 \pm 0.04$ & $-0.46 \pm 0.05$ & $<0.001$ \\
\hline $1-85$ days & & $-0.82 \pm-0.06$ & & $-0.60 \pm-0.06$ & $<0.001$ \\
\hline \multicolumn{6}{|l|}{ Symptom severity reflective (VAS) } \\
\hline days $2-29$ & $61.2 \pm 1.20$ & $-19.7 \pm 1.52$ & $59.80 \pm 1.19$ & $-12.7 \pm 1.50$ & $<0.001$ \\
\hline days $2-85$ & & $-25.7 \pm 1.64$ & & $-18.0 \pm 1.61$ & $<0.001$ \\
\hline Patient's evaluation of response (day 85) & NA & $2.70 \pm 0.10$ & NA & $3.11 \pm 0.10$ & $<0.001$ \\
\hline
\end{tabular}

LS, least significant. means, SEM (SE of the LS means) are obtained from an ANOVA model with treatment and site effects. NA, not applicable.

\section{REFERENCES}

1. Desloratadine (AERIUS ${ }^{\circledR}$ ). Prescribing information. Kenilworth, NJ: Schering Plough Corporation; 2005.

2. The ARIA/EAACI criteria for antihistamines: an assessment of the efficacy, safety and pharmacology of desloratadine. Allergy. 2004;59(77 Suppl):4-16.

3. Canonica GW, Tarantini F, Compalati E, Penagos M. Efficacy of desloratadine in the treatment of allergic rhinitis: a meta-analysis of randomized, double-blind, controlled trials. Allergy. 2007;62(4):359-366.

4. Bousquet J, Van Cauwenberge P, Khaltaev N. Allergic rhinitis and its impact on asthma. J Allergy Clin Immunol. 2001;108(5 Suppl):S147-S334.

5. Bousquet J, Khaltaev N, Cruz AA, Denburg J, Fokkens WJ, et al. Allergic rhinitis and its impact on asthma (ARIA) 2008 update (in collaboration with the World Health Organization, GA(2)LEN and AllerGen). Allergy. 2008;86(63 Suppl):8-160.

6. Bachert C, Bousquet J, Canonica GW, Durham SR, Klimek L, et al. Levocetirizine improves quality of life and reduces costs in long-term management of persistent allergic rhinitis. J Allergy Clin Immunol. 2004;114(4):838-844.

7. Fantin S, Maspero J, Bisbal C, Agache I, Donado E, et al. A 12-week placebo-controlled study of rupatadine $10 \mathrm{mg}$ once daily compared with cetirizine $10 \mathrm{mg}$ once daily, in the treatment of persistent allergic rhinitis. Allergy. 2008;63(7):924-931.
8. Nayak AS, Schenkel E. Desloratadine reduces nasal congestion in patients with intermittent allergic rhinitis. Allergy. 2001;56(11):1077-1180.

9. Berger WE, Schenkel EJ, Mansfield LE. Safety and efficacy of desloratadine $5 \mathrm{mg}$ in asthma patients with seasonal allergic rhinitis and nasal congestion. Ann Allergy Asthma Immunol. 2002;89(5):485-491.

10. Simons FE, Prenner BM, Finn A Jr. Efficacy and safety of desloratadine in the treatment of perennial allergic rhinitis. J Allergy Clin Immunol. 2003;111(3):617-622.

11. Kim K, Sussman G, Hebert J, Lumry W, Lutsky B, Gates D. Desloratadine therapy for symptoms associated with perennial allergic rhinitis. Ann Allergy Asthma Immunol. 2006;96(3):460-465.

12. Bousquet J, Bachert C, Canonica GW, Mullol J, Van Cauwenberge P, et al. Efficacy of desloratadine in intermittent allergic rhinitis A GA²LEN study. Allergy. 2009;64(10):1516-1523.

13. International Consensus Report on Diagnosis and Management of Rhinitis. International Rhinitis Management Working Group. Allergy. 1994;49(19 Suppl):1-34.

14. Heinzerling L, Frew AJ, Bindslev-Jensen C, Bonini S, Bousquet J, et al. Standard skin prick testing and sensitization to inhalant allergens across Europe-a survey from the GALEN network. Allergy. 2005;60(10): $1287-1300$.

15. Bousquet PJ, Combescure C, Neukirch F, Klossek JM, Mechin H, Daures JP, Bousquet J. Visual analog scales can assess the severity of 
rhinitis graded according to ARIA guidelines. Allergy. 2007;62 (4):367-372.

16. Bousquet PJ, Fabbro-Peray P, Janin N, Annesi-Maesano I, Neukirch F, Daures JP, Bousquet J. Pilot study assessing the impact of smoking on nasal-specific quality of life. Allergy. 2004;59(9):1015-1016.

17. Bousquet J, Neukirch F, Bousquet PJ, Gehano P, Klossek JM, Le Gal M, Allaf B. Severity and impairment of allergic rhinitis in patients consulting in primary care. J Allergy Clin Immunol. 2006;117(1):158-162.

18. Bousquet J, Lund VJ, Van Cauwenberge P, Bremard-Oury C, Mounedji N, Stevens MT, El-Akkad T. Implementation of guidelines for seasonal allergic rhinitis: a randomized controlled trial. Allergy. 2003;58(8): 733-741.

19. Santos CB, Pratt EL, Hanks C, McCann J, Craig TJ. Allergic rhinitis and its effect on sleep, fatigue, and daytime somnolence. Ann Allergy Asthma Immunol. 2006;97(5):579-586.

20. Kay GG. The effects of antihistamines on cognition and performance. J Allergy Clin Immunol. 2000;105(6 Pt 2):S622-S627.

21. Scadding G. Predicting and establishing the clinical efficacy of a histamine h(1)-receptor antagonist: desloratadine, the model paradigm. Clin Drug Investig. 2005;25(3):153-164.
22. Terreehorst I, Hak E, Oosting AJ, Tempels-Pavlica Z, de Monchy JG, et al. Evaluation of impermeable covers for bedding in patients with allergic rhinitis. $N$ Engl J Med. 2003;349(3):237-246.

23. Bousquet J, Bachert C, Canonica GW, Mullol J, Van Cauwenberge $\mathrm{P}$, et al. Efficacy of desloratadine in persistent allergic rhinitis - a GA ${ }^{2}$ LEN study. Int Arch Allergy Immunol. 2010;153 (4):395-402.

24. Meltzer EO, Jalowayski AA, Vogt K, Iezzoni D, Harris AG. Effect of desloratadine therapy on symptom scores and measures of nasal patency in seasonal allergic rhinitis: results of a single-center, placebo-controlled trial. Ann Allergy Asthma Immunol. 2006;96(2):363-368.

25. Van Cauwenberge P, Watelet JB, Van Zele T, Bousquet J, Burney P, Zuberbier T. Spreading excellence in allergy and asthma: the GA2 LEN (Global Allergy and Asthma European Network) project. Allergy. 2005;60(7):858-864.

26. Frew AJ. GA2LEN-The Global Allergy and Asthma European Network. Clin Exp Allergy. 2005;35(2):122-125.

27. International Consensus Report on Diagnosis and Management of Rhinitis. International Rhinitis Management Working Group. Allergy. 1994; s49(19 Suppl):1-34. 